\title{
Prognostic factors for the safety and efficacy of retrograde laser lithotripsy: Data from a contemporary series of 155 consecutive patients with single and multiple lithiasis of the urinary tract
}

\author{
DIOMIDIS KOZYRAKIS ${ }^{1,2}$, GEORGIOS SOUKIAS ${ }^{1}$, DIMITRIOS KARAGIANNIS ${ }^{1}$, ANASTASIOS ZARKADAS ${ }^{1}$, \\ STEFANOS PERIKLEOUS ${ }^{1}$, STYLIANI-ELISSAVET CHATZISTAMOU ${ }^{1}$, ILIAS KATSAROS ${ }^{1}$, \\ KONSTANTINOS SKRIAPAS $^{3}$, MICHAEL LARDAS ${ }^{2}$, NIKOLAOS MERTZIOTIS ${ }^{2}$ and ZISIS KRATIRAS $^{1}$ \\ ${ }^{1}$ Department of Urology, 'Achillopoulio' General Hospital, Volos 32222; 'Department of Urology, 'Metropolitan' \\ General Hospital, Athens 15562; ${ }^{3}$ Department of Urology, General Hospital of Larisa, Larisa 41447, Greece
}

Received October 25, 2021; Accepted December 17, 2021

DOI: $10.3892 / \mathrm{etm} .2022 .11221$

\begin{abstract}
Treatment of large and multiple stones located in the ureter and/or the kidney may be challenging. The aim of the current study was to evaluate the results and complications of retrograde endoscopic lithotripsy for stones located in the urinary tract and to determine prognostic factors for treatment outcome. From April 2017 to March 2020, eligible patients for the active treatment of ureterolithiasis with or without concomitant nephrolithiasis $<20 \mathrm{~mm}$ were enrolled in the study. The prognostic factors for the stone free rate (SFR) after the 1st and subsequent sessions and overall complications were assessed. Patients were divided into single or multiple lithiasis groups (groups A and B respectively). A comparison between these two groups was then conducted. Overall, 237 stones were detected in 155 patients, representing a mean burden of 1.53 stone per patient. The mean total stone size was $14.7 \mathrm{~mm}$, the initial SFR was 80\% and the final SFR (after a mean of 1.23 session per patient) was $94.2 \%$. The rate of complications was $26.4 \%$. Multivariative analysis revealed that preoperative stenting and total stone size were independent
\end{abstract}

Correspondence to: Dr Diomidis Kozyrakis, Department of Urology, 'Metropolitan' General Hospital, 264 Mesogeion Avenue, Athens 15562, Greece

E-mail: dkozirakis@yahoo.gr

Abbreviations: SFR, stone free rate; SFR1, stone free rate after the first session; SFRf, stone free rate after any subsequent session(s) after the first; URL, ureteroscopicaly guided laser lithotripsy; ESWL, extracorporeal shock wave lithotripsy; RIRS, retrograde intrarenal surgery; PCNL, percutaneous nephrolithotripsy; EAU, European Association of Urology; AUA, American Urologic Association; UTI, urinary tract infection; HU, hounsfield units; SR, semirigid; F, flexible; SD, standard deviation; IQR, interqurtile range; djs, double j stent

Key words: ureterolithiasis, nephrolithiasis, multiple stones, lithotripsy, stone free prognostic factors of initial SFR, while no independent factors were determined for final SFR. Age, total size and stones in the lower calyx were independent factors for complications. In group A and B, 114 and 41 cases with solitary and multiple stones were included, respectively. Excluding operation time $(\mathrm{P}=0.002)$, no significant differences were recorded in terms of initial $(\mathrm{P}=0.255)$ and final SFR $(\mathrm{P}=0.056)$, hospital stay $(\mathrm{P}=0.308)$, mean number of treatments $(\mathrm{P}=0.757)$ and the rate of complications $(\mathrm{P}=0.218)$ between the two groups. In conclusion, retrograde endoscopic management of multiple lithiasis has a favorable outcome irrespective of stone location. Older patients with higher burdens and stones in the lower calyx should be treated with caution.

\section{Introduction}

Ureteroscopicaly guided laser lithotripsy (URL) is the treatment of choice for patients with ureterolithiasis (1,2). For large intrarenal stones percutaneous nephrolithotripsy (PCNL) is the mainstay of therapy in terms of stone free rates but at a cost of increased invasiveness (3). Smaller stones in the kidney can also be treated with ESWL or retrograde intrarenal surgery (RIRS) $(3,4)$. There is an increasing body of evidence that for intrarenal stones $\leq 20 \mathrm{~mm}$ flexible retrograde lithotripsy may yield results comparable with those of PCNL but with reduced risk of complications (5-7). Furthermore, for medium and small sized stones located in the kidney, the superiority of PCNL over RIRS in terms of stone removal and the rate of complications is in contention (5).

Some patients harbor multiple stones in the same collecting system and the ureteroscopic access to the proximal stone may be impeded by the distal one. Treatment of large and multiple stones located in the ureter and/or the kidney may be therefore a challenging procedure. Apart from equipment availability, several other factors play important roles in the success of treatment, such as the location, the number and the size of stones (2). Evidence regarding the optimal treatment of multiple ipsillateral lithiasis is limited and EAU and AUA Guidelines provide no clear recommendations $(3,4)$. However, for multiple ureterolithiasis, lithotripsy of as many 
stones as possible is a reasonable treatment. For stones located simultaneously in the ureter and the kidney, fragmentation of the ureteral counterpart is initially performed and when the patency of ureter has been achieved removal of renal stones is attempted in a separate session of PCNL or ESWL.

Considering the improvements in the technology of flexible scopes, we investigated whether multiple ureterolithotripsy or ureterolithotripsy plus RIRS in a single session might have the potential to safely and effectively treat patients. To address this issue, the results of multiple lithotripsy are compared to the standard of care (single ureteral lithotripsy).

\section{Materials and methods}

Study population. From April 2017 to March 2020, patients eligible for active treatment for ureterolithiasis or concomitant lithiasis of the ureter and the kidney were enrolled in the study and prospectively evaluated. Data regarding sex, age, history of urinary tract infection (UTI), laterality, number, size and location of stones, preoperative stenting, duration of operation, stone hardness (expressed in Hounsfield units-HU), use of semirigid (SR) and/or flexible (F) instruments, use of basket and access sheath were recorded and analyzed. End points were stone free rate (SFR) after a single and multiple treatments [SFR1 and SFR final (SFRf) respectively] and the rate of complications. The stone size was the maximum length of a solitary stone in CT scan or the sum of the maximum lengths in multiple lithiasis. The stone surface or the volume after $3 \mathrm{D}$ reconstruction might be more representative of the stone burden. However, the maximum length or the sum of maximum lengths have also been used in the literature to describe the stone burden $(6,8-10)$. In the cases with multiple stones the $\mathrm{HU}$ was the mean value of the $\mathrm{HU}$ of each one. The operation time was calculated from the time the patient received anesthesia till the removal of the scope. Complications were graded according to the Clavien system (11). Analysis of stone composition was performed only in younger stone formers and in recurrent disease; hence stone composition was not formally included in the analysis.

Treatment methods. Criteria for exclusion were the presence of renal stones only, the renal stones greater than $20 \mathrm{~mm}$, uncontrolled coagulopathy, pregnancy, staghorn calculi and active urinary tract infection. Previous treatment for lithiasis was not a criterion for exclusion. Prior to the operation all of the participants were informed about all the treatment options for single and multiple lithiasis. Detailed information was provided about results and complications of URL and RIRS, and patients signed the relevant informed consent. The methods of treatment meet the criteria of Helsinki declaration for human rights and the study was approved by the General Hospital of Volos' ethics committee (protocol number 3718/17-3-2017). Those who consented on the treatment had also the option to be included in the study. Single or multiple retrograde ureterolithotripsy was initially performed aiming to disintegrate all the ipsilateral stones. In patients with intrarenal stones, when the patency of the ureter had been achieved the treatment was proceeded with RIRS.

For ureteral stones a $8 / 9.5 \mathrm{~F}$ semirigid ureteroscope (Richard Wolf $\mathrm{GmbH}$ ) was used. Some stones in the proximal ureter and all the renal ones were fragmentized with a 4.9/7.95 F flexible ureteroscope (URFP6, Olympus). Lithotripsy was performed using a $30 \mathrm{~W}$ Litho Ho:YAG laser system (Quanta System), with 365 or 200 micron laser fibers. The system was adjusted in the dusting mode with frequency ranging from 5 to $15 \mathrm{~Hz}$ and energy between 0.2 and $2 \mathrm{~J}$ according to stone hardness. 10-12F access sheaths (Uropass, Gyrus Medical Ltd.) were used to facilitate the reinsertion of the flexible scope.

Larger stone fragments were removed with either a 1.8 or a 3F nitinol tipless basket (Ultra-Catch, Olympus Europa) and double $\mathrm{j}$ stent (djs) was left in place for 7-10 days upon suspicion for minor ureteral trauma. Should a more severe ureteral trauma was suspected the stent was left for 4-6 weeks. SFR was defined as no residual stone or a single stone $<3 \mathrm{~mm}$ detected in unenhanced low dose CT scan performed 3 months after the procedure. The stone type was not formally examined in all the patients. Stone examination with infrared spectroscopy was performed only in younger patients and in those with frequent recurrences.

Statistical analysis. Data were analysed using Stata ${ }^{\mathrm{TM}}$ (Version 13.0 MP, Stata Corporation). The Shapiro-Wilk test was performed to test for normal distribution of continuous factors and the relevant results are given as mean $( \pm$ SD) while not normally distributed variables are presented as median values [IQR]. Univariate and multivariate logistic regression analysis were applied in order to identify independent prognostic factors related to the outcomes. All tests were two-tailed and statistical significance was established at $5 \%(\mathrm{P}<0.05)$.

\section{Results}

Patient characteristics. Overall 237 stones were detected in 155 patients who consented on for both the proposed treatment and their inclusion in the study too, representing a mean burden of 1.5 stone per patient. Patients' characteristics are presented in Table I.

For the total group of patients the mean age was 58.02 years, with a limited number of female participants in the study (79 males vs. 76 females, ratio 1.04:1). 30 cases had a history of previous treatment. With a median total stone size of $12 \mathrm{~mm}$ the SFR1 of a single procedure was $80 \%$. After a mean of 1.23 procedures per patient the SFRf was $94.2 \%$. The overall complications' rate was $26.4 \%$. Apart from lower urinary tract symptoms (LUTS) the list of complications included fever, hematuria, UTI with or without hospitalization, sepsis, ureteral trauma (but without ureteral avulsion events) and ureteral stenosis. Acute myocardial infarction was postoperatively diagnosed in one patient. Overall 15 patients experienced Clavien grade 3-4 complications (9.7\%).

Total group analysis. In univariative analysis (Table II), total number of stones, presence of stone in the lower calyx, total stone size and preoperative stenting were factors correlated with SFR1. Total stone number, total stone size, number of stones in the ureter and kidney, presence of stones in the lower calyx and total stone size were correlated with the SFRf. In respect to the complications only age and total stone size were significant factors. 
Table I. Patient characteristics.

\begin{tabular}{|c|c|c|c|c|}
\hline Factor & Total group $(n=155)$ & Group A $(n=114)$ & Group B $(n=41)$ & P-value \\
\hline Age in years $( \pm S D)$ & $58.02(13.69)$ & $57.3(14.31)$ & $59.9(11.72)$ & 0.3075 \\
\hline Male sex, n (\%) & $79(51)$ & $55(48.25)$ & $21(51.22)$ & 0.856 \\
\hline Laterality (Rg=left), n (\%) & $75(48.4)$ & $54(47.37)$ & $21(51.22)$ & 0.718 \\
\hline HU (IQR) & $780[620-928]$ & $816[640-1050]$ & $771[544-910]$ & 0.156 \\
\hline Type of intervention, $\mathrm{n}(\%)$ & & & & $<0.001$ \\
\hline F only & $5(3.2)$ & $5(4.39)$ & $0(0.00)$ & \\
\hline SR only & $93(60)$ & $84(73.68)$ & $9(21.95)$ & \\
\hline $\mathrm{SR}+\mathrm{F}$ & $57(36.8)$ & $25(21.93)$ & $32(78.05)$ & \\
\hline Total size in mm, median (IQR) & $12[8-17]$ & $10[7-15]$ & $22[16-32]$ & $<0.001$ \\
\hline Total number of stones (mean) & $237(1.52)$ & $114(1)$ & $123(3)$ & 0.034 \\
\hline Number of ureter stones (mean) & $192(1.24)$ & $114(1)$ & $80(1.95)$ & NA \\
\hline Number of kidney stones (mean) & $44(0.28)$ & $0(0 \%)$ & $44(1.07)$ & NA \\
\hline Stone in lower calyx, $\mathrm{n}(\%)$ & $7(4.5 \%)$ & $0(0 \%)$ & $7(17.1 \%)$ & NA \\
\hline Stent preoperatively, n (\%) & $90(58.1)$ & $66(57.9)$ & $24(58.5)$ & $>0.999$ \\
\hline Stent postoperatively, n (\%) & $127(81.9)$ & $93(81.6)$ & $34(82.3)$ & $>0.999$ \\
\hline Access Sheath, n (\%) & $42(27.1)$ & $17(14.9)$ & $25(60.9)$ & $<0.001$ \\
\hline Use of basket, n (\%) & $69(44.5)$ & $41(35.9)$ & $28(68.3)$ & $<0.001$ \\
\hline Operation time, mins (IQR) & $60[45-95]$ & $57.5[42-90]$ & $88[55-105]$ & 0.002 \\
\hline Hospital Stay days (IQR) & $1[1-2]$ & $1[1-2]$ & $1[1-3]$ & 0.308 \\
\hline SFR1, n (\%) & $124(80)$ & $94(82.5)$ & $30(73.2)$ & 0.255 \\
\hline Chemolysis, n (\%) & $4(2.6)$ & $1(0.9)$ & $3(7.3)$ & 0.057 \\
\hline Complication, n (\%) & $41(26.4)$ & $27(23.7)$ & $14(34.1)$ & 0.218 \\
\hline Clavien grade $3-4, \mathrm{n}(\%)$ & $15(9.7)$ & $10(8.8)$ & $5(12.2)$ & 0.112 \\
\hline Sepsis, n (\%) & $11(7.1)$ & $8(7)$ & $3(7.3)$ & 0.435 \\
\hline Number of treatments, $\mathrm{n}(\%)$ & & & & 0.757 \\
\hline 1 & $128(82.6)$ & $95(83.3)$ & $33(80.5)$ & \\
\hline 2 & $19(12.3)$ & $14(12.3)$ & $5(12.2)$ & \\
\hline 3 & $8(5.1)$ & $5(4.4)$ & $3(7.3)$ & \\
\hline Total number of treatments (mean per patient) & $186(1.23)$ & $138(1.21)$ & $52(1.27)$ & \\
\hline SFRf, n (\%) & $146(94.2)$ & $110(96.5)$ & $36(87.8)$ & 0.056 \\
\hline
\end{tabular}

F, flexible; IQR, interquartile range; NA, non available; Rg, reference group; SD, standard deviation; HU, Hounsfield units; SFR1, stone free rate in one session; SFRf, final stone free rate (after all sessions); SR, semirigid.

Multivariative logistic regression analysis (Table III) revealed that preoperative stent placement and total stone size were independent prognostic factors for SFR1. For the end point of SFRf no independent prognostic factor was detected. Age, stone in the lower calyx and total stone size were independently associated with increased complications.

Comparative analysis. In 41 cases multiple stones were detected with a mean burden of 3 stones per patient (group B). The control group consisted of 114 cases with single ureterolithiasis (group A). The majority of patients of group B were treated with the combined SR and F endoscopic lithotripsy aided by the placement of access sheath while for group A SR endoscopy was more frequently implicated. The median lithiasic burden was higher ( 22 vs. $10 \mathrm{~mm}, \mathrm{P}<0.001)$ and the duration of intervention longer ( 88 vs. $57.5 \mathrm{~min}, \mathrm{P}=0.002$ ) in group B compared with group A respectively. However, the median duration of hospital stay was similar (one day for each group). After one lithotripsy procedure the SFR1 was similar for both groups $(82.5$ and $73.2 \%$ for group A and B respectively) $(\mathrm{P}=0.255)$.

Likewise, neither the outcome of SFRf (96.5\% vs. 87.8\%) nor the mean number of procedures per patient (1.21 vs. 1.27) for the group B and A respectively were statistical significant different (Table I). Of the 5 treatment failures of group B, two hosted stones in the lower calyx.

\section{Discussion}

ESWL is a safe and effective method of treating urinary stones of small and medium size, particularly those located in the upper urinary tract (8). However, for lithiasic burdens $>20 \mathrm{~mm}$ located in the kidney the results of ESWL are compromised and the reported stone free rates are approximately $50-55 \%(9,12)$. Likewise, retrograde endoscopy and laser lithotripsy, either with flexible or semirigid scope, is the standard of care for 
Table II. Univariate logistic regression models for the identification of factors related to SFR1, SFRf and overall complications for the total group of patients.

\begin{tabular}{|c|c|c|c|c|c|c|c|c|c|}
\hline \multirow[b]{2}{*}{ Outcome } & \multicolumn{3}{|c|}{ SFR1 } & \multicolumn{3}{|c|}{ SFRf } & \multicolumn{3}{|c|}{ Complications } \\
\hline & OR & $95 \% \mathrm{CI}$ & P-value & OR & $95 \% \mathrm{CI}$ & P-value & OR & $95 \% \mathrm{CI}$ & P-value \\
\hline $\operatorname{Sex}(\mathrm{Rg}=$ females $)$ & 0.75 & $0.34-1.65$ & 0.470 & 0.76 & $0.19-2.93$ & 0.687 & 1.32 & $0.65-2.71$ & 0.444 \\
\hline Age & 1.03 & $0.99-1.06$ & 0.060 & 1.002 & $0.95-1.05$ & 0.944 & 1.03 & $1.003-1.06$ & 0.027 \\
\hline Laterality $(\mathrm{Rg}=\mathrm{left})$ & 0.25 & $0.10-0.60$ & 0.203 & 0.45 & $0.11-1.86$ & 0.269 & 0.66 & $0.32-1.35$ & 0.251 \\
\hline Total no. of stones & 1.49 & $1.08-2.06$ & 0.015 & 2.10 & $1.35-3.26$ & 0.001 & 1.35 & $0.99-1.84$ & 0.060 \\
\hline No. of ureter stones & 1.57 & $0.99-2.49$ & 0.055 & 1.94 & $1.12-3.37$ & 0.018 & 1.47 & $0.93-2.31$ & 0.096 \\
\hline No. of kidney stones & 1.49 & $0.93-2.38$ & 0.097 & 2.14 & $1.21-3.79$ & 0.009 & 1.29 & $0.81-2.04$ & 0.277 \\
\hline Stone in the lower calyx $(\mathrm{Rg}=\mathrm{no})$ & 5.97 & $1.26-28.3$ & 0.024 & 8.06 & $1.32-49.1$ & 0.024 & 1.12 & $0.21-5.99$ & 0.897 \\
\hline Total stone size & 1.08 & $1.03-1.12$ & 0.001 & 1.12 & $1.05-1.19$ & 0.001 & 1.07 & $1.03-1.12$ & 0.001 \\
\hline Stent preoperatively $(\mathrm{Rg}=\mathrm{no})$ & 2.44 & $1.01-5.89$ & 0.046 & 1.48 & $0.36-6.13$ & 0.592 & 1.35 & $0.65-2.82$ & 0.419 \\
\hline
\end{tabular}

CI, confidence interval; OR, odds ratio; Rg, reference group; SFR1, stone free rate in one session; SFRf, final stone free rate (after all sessions).

Table III. Multivariate logistic regression models for the identification of independent factors related to SFR 1 and complications. A, SFR1

\begin{tabular}{lcccrr}
\hline Factor & OR & Std. Err. & $\mathrm{z}$ & P>lzl & 95\% CI \\
\hline Stent preoperatively & 0.2799494 & 0.1657261 & 2.15 & 0.032 & $0.0877365-0.8932622$ \\
Total stone size & 0.9451793 & 0.0229961 & -2.32 & 0.020 & $0.9011655-0.9913428$ \\
\hline
\end{tabular}

B, Complications

\begin{tabular}{llcrrr}
\hline Factor & \multicolumn{1}{c}{ OR } & Std. Err. & $\mathrm{z}$ & P>lz| & $95 \%$ CI \\
\hline Age & 0.032445 & 0.0158132 & -2.82 & 0.037 & $1.001912-1.063908$ \\
Stone in the lower calyx & 0.094723 & 0.1072088 & -2.08 & 0.042 & $0.0103053-0.8706735$ \\
Total stone size & 1.10058 & 0.0274499 & -3.84 & $<0.001$ & $1.048073-1.155717$ \\
\hline
\end{tabular}

CI, confidence interval; OR, odds ratio; SFR1, stone free rate in one session; Std. Err., standard error.

ureterolithiasis but it is still an option for intrarenal stones greater then $>20 \mathrm{~mm}(3)$. In one of the most recent systematic reviews, the SFR was inferior but the hospital staying was shorter following flexible RIRS compared with PCNL (5). In the settings of large stones of the upper tract therefore, PCNL is the standard of care but at a cost of higher invasiveness and increased risk for complications particularly if multiple punctures are required $(13,14)$.

In a high percentage of lithiasic patients, multiple stones may be encountered. In our study 41 out of 155 patients were diagnosed with multiple lithiasis corresponding to the rate of $26.4 \%$. This figure is similar to the rates reporting in other series ranging between 20 and $25 \%$ (9). In the settings of multiple lithiasis, URS with or without RIRS could be considered for treating these patients $(14,15)$. In a single arm analysis comprising patients with multiple stones two out of three cases $(66.7 \%)$ were rendered stone free one month after a single flexible retrograde lithotripsy (16). Takazawa et al showed that for a mean lithiasic burden of $21.5 \mathrm{~mm}$, a figure similar to that of group B of the present study, the SFRs were 80 and $92 \%$ after the first and the second procedure respectively (8). In another report comprising patients with multiple nephriolithiasis, the SFR was as high as $100 \%$ for a total size of $<20 \mathrm{~mm}$ compared with $84 \%$ in patients with larger sum of lengths (9). For stones $<20 \mathrm{~mm}$ Breda et al reported a SFR of 79 and $100 \%$ after one and two procedures respectively (10). Among our patients of group B, with a median size of $22 \mathrm{~mm}$ and a median stone number of 3 , the initial and final SFR was 73.2 and $87.8 \%$ respectively which compare well with the aforementioned reports.

As it is shown in Table III, in mulivariative analysis the total stone size and the presence of ureteral stent were independent prognostic factors for SFR1. This means that neither the number nor the location of stones in the collecting system had a significant influence on the results of the first lithotripsy and the physician should not be discouraged from proposing 
and offering retrograde lithotripsy to the patients with multiple and/or large stones, particularly to those with pre-stented ureters. It could also be supported that the modern lithotripsy scopes and laser devices enables for an effective and safe treatment of multiple stones and of larger lithiasic burdens compared with the past.

Moreover, for the end point of SFRf no significant predictive factors were revealed. It seems that the first lithotripsy enhanced the results of subsequent or auxiliary session maximizing the stone clearance effect. The djs, which was placed in almost $60 \%$ of the patients before the first lithotripsy, was then present in all the ureters before the second procedure, facilitating thus the outcome of the reoperation. Although the lower calyx lithiasis was correlated with the SFRs in univariative analysis, this variant proved to be an independent prognostic factor only for complications in multivariative settings. In other words, the presence of stones in the lower calyx was not independently associated with the stone clearance. It seems that the modern flexible endoscopes and the more sophisticated laser devices enables for favorable SFR outcomes of lower pole lithiasis. The aforementioned reasons, combined with the limited rate of patients $(20 \%)$ registered for reoperation, could be the plausible explanations for the lack of significant prognostic factors for the end point of SFRf.

Increased age, large total lithiasic burden and/or stones in the lower calyx were independently associated with increased complications. It seems that older patients are more vulnerable, compared with the younger population. Older patients are prone in developing postoperative LUTS, infections and fever probably due to their aging urinary tract system and the increased volume of the prostate in male patients. Perhaps in the elderly the previous exposure to bacteria and the delayed mucosal healing after a minor trauma are both related with increased complications rate. Therefore, careful manipulations of the instruments, avoidance of high pressure irrigation and minimum operation time are issues that could be followed for diminishing complications. These patients should be informed about the increased odds of complications before signing the informed consent and should be treated with caution irrespectively of the number of detected stones or the presence of double $\mathrm{j}$ stent.

Our report is one of the few studies to investigate prognostic factors for the outcomes of retrograde lithotripsy. Similar to our findings, Lee et al, by using the threshold of $100 \mathrm{~mm}^{2}$, revealed that the cumulative stone burden was an independent prognostic factor for the one-month SFR. They also did not reveal any correlation with the stone number or their location in the urinary tract (16). Lai et al reported lower rates of complications in pre-stented patients with renal calculi (15), a finding that is not confirmed in the present study though.

At the second stage of our study, emphasis was given on the comparison of the outcomes of multiple lithotripsy (group B) vs. single URL as the standard of care (group A) (Table II). As expected, the median duration of operation was longer for multiple lithiasis by approximately $30 \mathrm{~min}$. However, this duration $(88 \mathrm{~min}$ ) is reasonable considering that in other reports with similar or lower lithiasic burden the mean operative time ranges between 72 and $112 \min (8,15,16)$.
More importantly, we sought to determine whether the prolonged operation time and the need for multiple re-insertions of instruments may increase the rate or severity of complications. This hypothesis is not confirmed in the present analysis. The overall complications' rate were higher for group B compared to A (34.15\% vs. $23.7 \%$ respectively) but it was not of statistical significance $(\mathrm{P}=0.218)$. Likewise, no impact on duration of hospital staying was recorded for the group of multiple stones (median value: one day for either group A or $\mathrm{B}, \mathrm{P}=0.3081$ ). Although logistic regression analysis was not feasible for severe Clavien grade $\geq 3$ complications and for sepsis, the rate of this outcome was also similar between the groups confirming the safety of multiple lithotripsy. In addition, ureteral stenosis and trauma were also rarely encountered (in one patient of group B and in two others of group A).

Reviewing the literature, the rate of adverse effect range between 5\% $(14,16)$ and $23 \%(12,15)$ for retrograde lithotripsy. Comparatively, in the present study higher rates of complications (23.7 and $34.1 \%$ for group A and B respectively) are revealed. These finding could be attributed mainly to the inclusion of lower urinary tract symptoms (LUTS) in the list of complications. Should LUTS are excluded from analysis the complication rates for group A and B were much lower (22 and $18 \%$ respectively).

Compared to single fragmentation, the multiple lithotripsy procedure yielded inferior results for the end points of initial and final SFR respectively. These differences are however not of statistical significance ( $\mathrm{P}=0.255$ and 0.056 respectively) indicating that the outcomes are not compromised in group B. The total number of procedures per patient were also similar between the two groups (1.21 vs. 1.27 for group A and B respectively, $\mathrm{P}=0.757$ ) which also confirms the merit of multiple lithotripsy. As it was mentioned previously, in multivariative analysis the total stone size was not an independent prognostic factor for SFRf. In other words, although flexible RIRS is not considered the standard of care for large stones, it could still be an attractive treatment for multiple stones including the renal ones, indicating a shift of the treatment paradigm from PCNL towards an even less invasive procedure for stones with sizes even larger than $20 \mathrm{~mm}$.

We used access sheath in the majority of patients with large stones; it facilitated the insertion of the flexible scopes, in removal of stone fragments and to shortening of the operation time. More importantly, access sheath may prevent excessive increase of intrarenal pressure within the pelvis during prolonged fragmentation. In a multicenter trial, concerns were raised about ureteral injury after access sheath placement in the settings of large lithiasic burden (17) but they were not confirmed in the present study. Injury of the ureter was recorded in 2 patients; in only one of them the injury was associated with placement of access sheath. In another patient, ureteral stenosis was diagnosed shortly after the removal of djs and a ureteral stent was reinserted. In this patient access sheath was not used though. It should be emphasized however that the comparison between patients with single or multiple lithiasis should be made with caution considering that the preoperative characteristics regarding the stone size and location were not similar between A and B group. The number of patients registered in each group was also dissimilar raising some concerns about the risk of bias following the comparison 
of the postoperative results. Moreover, the length of the stone might not be the ideal index of the stone burden, considering that this parameter is better represented by the stone surface or the volume after 3D reconstructions of the CT slices. Nevertheless, several authors $(6,8-10)$ have clearly defined and used the sum of maximum diameters of the stones as a surrogate for the total stone burden. This methodology was also applied in the present manuscript too. We also acknowledge that a larger study population particularly in the group of multiple lithiasis would be preferable for safer and more robust outcomes to be extracted. All the aforementioned are considered as limitations of the study.

Despite the limitations of the study, by using logistic regression analysis, plausible prognostic factors for the likelihood of stone clearance and for the odds of complications were revealed. Older patients with higher burdens and stones in the lower calyx should be treated with caution. It was also shown that for sizes up to $22 \mathrm{~mm}$, the performance of URL with or without RIRS is optimal against multiple stones irrespectively to their location, albeit more scientific evidence is required. Patients with larger burdens could also experience benefit though, by reducing the need for auxiliary treatments or enhancing the outcome of any subsequent procedure.

\section{Acknowledgements}

Not applicable.

\section{Funding}

No funding was received.

\section{Availability of data and materials}

The datasets used and/or analyzed during the current study are available from the corresponding author on reasonable request.

\section{Authors' contributions}

DKo and ZK confirm the authenticity of all the raw data. DKo and GS conceived the study, and ZK and KS contributed to the study design. DKa and AZ performed the experiments and collected the raw data. SP, SEC, IK and NM contributed equally to the analysis and interpretation of the results. ML participated in the analysis and interpretation of data. KS, $\mathrm{ML}$ and NM wrote and reviewed the manuscript. DKo, ML and ZK performed a literature review. All authors read and approved the final manuscript.

\section{Ethics approval and consent to participate}

Prior to any treatment, all participants were informed about the treatment options available for single and multiple lithiasis. Detailed information was provided about the results and complications of retrograde ureterolithotripsy and retrograde intrarenal surgery. Patients who accepted the details provided written informed consent. The methods of treatment meet the criteria of Helsinki declaration for human rights and the study was approved by the General Hospital of Volos Ethics Committee (protocol no. 3718/17-3-2017).

\section{Patient consent for publication}

Not applicable.

\section{Competing interests}

The authors declare that they have no competing interests.

\section{References}

1. Bagley D: Expanding role of ureteroscopy and laser lithotripsy for treatment of proximal ureteral and intrarenal calculi. Curr Opin Urol 12: 277-280, 2002.

2. Perez Castro E, Osther PJ, Jinga V, Razvi H, Stravodimos KG, Parikh K, Kural AR and de la Rosette JJ; CROES Ureteroscopy Global Study Group: Differences in ureteroscopic stone treatment and outcomes for distal, mid-, proximal, or multiple ureteral locations: The clinical research office of the endourological society ureteroscopy global study. Eur Urol 66: 102-109, 2014.

3. Turk C, Skolarikos A, Neisius A, Petrik A, Seitz C, Thomas K; Guidelines Associates, Donaldson JF, Drake T, Grivas $\mathrm{N}$ and Ruhayel Y: EAU Guidelines on Urolithiasis. Available from: http://uroweb.org/wp-content/uploads/ EAU-Guidelines-on-Urolithiasis-2019-large-text.pdf.

4. Assimos D, Krambeck A, Miller NL, et al: Surgical Management of Stones. AUA/Endourological Society Guideline 2016 accessed in https://www.auanet.org/guidelines/ kidney-stones-surgical-management-guideline.

5. Zhu M, Wang X, Shi Z, Ding M, Fan D, Wang X and Jiang R: Comparison between retrograde intrarenal surgery and percutaneous nephrolithotripsy in the management of renal stones: A meta-analysis. Exp Ther Med 18: 1366-1374, 2019.

6. Hyams ES, Munver R, Bird VG, Uberoi J and Shah O: Flexible ureterorenoscopy and holmium laser lithotripsy for the management of renal stone burdens that measure 2 to $3 \mathrm{~cm}$ : A multi-institutional experience. J Endourol 24: 1583-1588, 2010.

7. Xu C, Song RJ, Jiang MJ, Qin C, Wang XL and Zhang W: Flexible ureteroscopy with holmium laser lithotripsy: A new choice for intrarenal stone patients. Urol Int 94: 93-98, 2015.

8. Takazawa R, Kitayama S and Tsujii T: Single-session ureteroscopy with holmium laser lithotripsy for multiple stones. Int $\mathbf{J}$ Urol 19: 1118-1121, 2012.

9. Alkan E, Ozkanli O, Avci E, Turan M, Başar MM, Acar O and Balbay MD: Effectiveness of flexible ureterorenoscopy and laser lithotripsy for multiple unilateral intrarenal stones smaller than $2 \mathrm{~cm}$. Adv Urol 2014: 314954, 2014.

10. Breda A, Ogunyemi O, Leppert JT and Schulam PG: Flexible ureteroscopy and laser lithotripsy for multiple unilateral intrarenal stones. Eur Urol 55: 1190-1196, 2009.

11. Clavien PA, Barkun J, de Oliveira ML, Vauthey JN, Dindo D, Schulick RD, de Santibañes E, Pekolj J, Slankamenac K, Bassi C, et al: The Clavien-Dindo classification of surgical complications: Five-year experience. Ann Surg 250: 187-196, 2009.

12. Ozgor F, Kucuktopcu O, Ucpinar B, Gurbuz ZG, Sarilar O, Berberoglu AY, Baykal M and Binbay M: Is there a difference between presence of single stone and multiple stones in flexible ureterorenoscopy and laser lithotripsy for renal stone burden $<300$ mm2? Int Braz J Urol 42: 1168-1177, 2016.

13. Lawler AC, Ghiraldi EM, Tong C and Friedlander JI: Extracorporeal shock wave therapy: Current perspectives and future directions. Curr Urol Rep 18: 25, 2017.

14. Herrera-Gonzalez G, Netsch C, Oberhagemann K, Bach T and Gross AJ: Effectiveness of single flexible ureteroscopy for multiple renal calculi. J Endourol 25: 431-435, 2011.

15. Lai D, Chen M, He Y and Li X: Simultaneous retrograde intrarenal surgery for ipsilateral asymptomatic renal stones in patients with ureteroscopic symptomatic ureteral stone removal. BMC Urol 15: 22, 2015.

16. Lee SH, Kim TH, Myung SC, Moon YT, Kim KD, Kim JH, Kwon JK and Chang IH: Effectiveness of flexible ureteroscopic stone removal for treating ureteral and ipsilateral renal stones: A single-center experience. Korean J Urol 54: 377-382, 2013.

17. Loftus CJ, Ganesan V, Traxer O, Schold JD, Noble M, Sivalingam S, Muruve N and Monga M: Ureteral wall injury with ureteral access sheaths: A randomized prospective trial. J Endourol 34: 932-936, 2020. 